\title{
MIXED CONVECTIVE FLOW WITH VARIABLE VISCOSITY AND VARIABLE THERMAL CONDUCTIVITY IN A CHANNEL IN THE PRESENCE OF FIRST ORDER CHEMICAL REACTION WITH HEAT GENERATION OR ABSORPTION
}

\author{
Shobha K $C^{1}$, Mahadev Biradar ${ }^{2}$, Patil Mallikarjun $B^{1}$ \\ ${ }^{1}$ Department of Studies and Research in Mathematics, Tumkur University, Tumakuru \\ Karnataka, India \\ ${ }^{2}$ Department of Mathematics, Basaveshwar Engineering College, Bagalkot, Karnataka, India \\ shobhakc1995@gmail.com,mbp1007@yahoo.com
}

Received: 26 June 2020; Accepted: 3 February 2021

\begin{abstract}
A study has been made on the flow and heat transfer of a viscous fluid in a vertical channel with first order chemical reaction and heat generation or absorption assuming that the viscosity and thermal conductivity are dependent on the fluid temperature. The temperature of the walls is maintained constant. Under these assumptions, the governing balance equations of mass, momentum and energy are formulated. The dimensionless forms of the governing equations are coupled and non-linear, which cannot be solved analytically and therefore require the use of the Runge-Kutta fourth order along with shooting technique. Graphs for velocity and temperature under different values of parameters involved are plotted and discussed. The skin friction and Nusselt number on the channel walls are also computed and discussed. Furthermore, the investigation found that variable viscosity and variable thermal conductivity enhance the velocity and temperature of the flow.
\end{abstract}

MSC 2010: 34B08, 65L06, 76D05

Keywords: mixed convection, variable viscosity, variable thermal conductivity, first order chemical reaction, heat generation/absorption

\section{Introduction}

In the past few decades, considerable interest has been given to study the flow of an incompressible viscous fluid over a continuous moving solid surface which has important applications in a variety of manufacturing processes. Such processes are hot rolling, wire drawing, spinning of filaments, metal extrusion, crystal growing, continuous casting, glass fiber production, and paper production. There is a major influence of temperature dependent viscosity and temperature dependent thermal conductivity on the flow and heat transfer along with the effect of heat generation and absorption. 
In the literature of Bejan [1] and Umavathi [2], they extensively studied the natural convection and heat transfer in channels and also considered the flow in a porous medium. Many researchers have included the effect of chemical substances present in the flow reaction. Muthucumaraswamy and Ganesan [3] reported that the number of time steps to reach the steady-state depends strongly on the chemical reaction parameter. The reaction reduces the local concentration, thus increasing its concentration gradient and its flux. Umavathi et al. [4] studied mixed convection flow in a vertical porous stratum with asymmetric wall heating conditions. The effects of the Hartmann number, chemical reaction, variable viscosity, dimensionless wall mass transfer coefficient, Schmidt number, thermophoresis parameter and the dimensionless heat generation/absorption coefficient on the flow and heat transfer have been studied by Seddeek [5] and they have used the finite element method to solve the governing equations.

In many situations like in the field of metallurgy and chemical engineering, the physical properties like viscosity and thermal conductivity need not be constants they varies with the temperature. Herwig and Wickern [6] and Pop et al. [7] and Abel et al. [9] have analysed the flow and heat transfer with varying viscosity. The variable thermal conductivity effect was considered by Chiam [8]. When the variable viscosity effect is included the flow characteristics, may change substantially compared to constant viscosity assumption. For lubricating fluids, heat generated by internal friction and the corresponding rise in the temperature affects the viscosity of the fluid and so that the fluid viscosity no longer be assumed constant. This was shown by Gary et al. [10] and Mehta [11]. The influence of variable property effects on the induced mass flow rate and on the size of the recirculation region in a channel formed by an isothermal plate, and an adiabatic plate has been analysed by Zamora and Hernández [12, 13].

The flow of fluid with internal heat generation/absorption is of great experimental as well as theoretical importance. The volumetric heat generation/absorption term exerts a strong effect on the heat transfer and flow when the temperature difference is significantly high. Heat generation effects may alter the temperature distribution; consequently, the particle deposition rate in nuclear reactors, electronic chips and semiconductor wafers. Patil Mallikarjun et al. in [16] showed that the effect of the heat generation coefficient is to decrease the velocity and increase temperature.

In many hydrometallurgical industries and chemical engineering processes, chemical reaction occurs between a foreign mass and the working fluid. There are many transport processes governed by the combined action of buoyancy forces due to both thermal and mass diffusion in the presence of chemical reaction effects. A first order chemical reaction is one in which the rate of the reaction is directly proportional to the concentration of the reactant species. Chamka [17] analysed the heat and mass transfer by steady flow of electrically conducting fluid on a uniformly moving vertical surface in the presence of first order chemical reaction. Fully developed laminar mixed convection flow in a vertical channel in the presence of first order chemical reactions has been studied Prathap Kumar et al. [18]. 
Patil Mallikarjun et al. have studied the effect of heat generation and absorption along with other effects such as first order chemical reaction [19].

With this above mentioned literature survey, in this paper the physical properties of the fluid like viscosity and thermal conductivity of the fluid are taken to be variables and vary as exponential functions of temperature as taken by Umavathi [20] to analyse the mixed convective flow in the channel in the presence of first order chemical reaction with heat generation or absorption. The governing equations are solved by using the Runge-Kutta fourth order shooting method. Velocity and temperature profiles for different values of physical parameters are analysed graphically.

\section{Mathematical formulation}

Let us consider the steady flow of a viscous fluid in a vertical channel as described in Figure 1. The rectangular coordinate system is fixed at the middle of the channel with parallel plates separated by a distance $2 b$ so that the width of the channel lies in $-b \leq Y \leq b$. Flow of the fluid in the channel is due to buoyancy forces. The left and right walls are maintained at temperatures $T_{1}$ and $T_{2}$ respectively. For the velocity boundary conditions, no slip condition is applied. Since the plates of the channel are of infinite length in $X$-direction, the velocity component is $U(Y)$ and is only along $X$-axis.

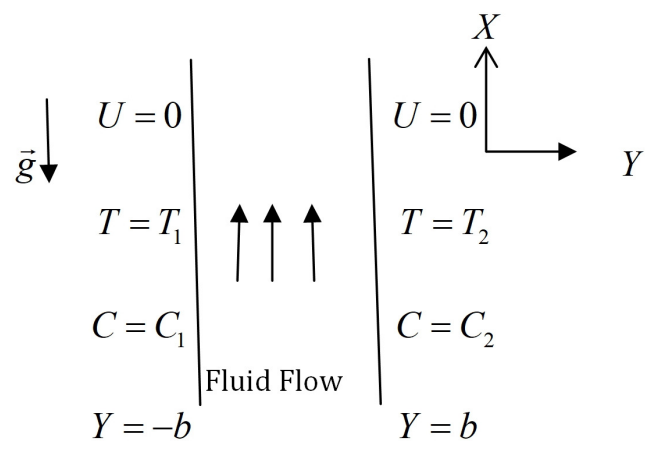

Fig. 1. Physical configuration of the model and coordinate systems

The physical properties of the fluid are assumed to be constant except density, viscosity and thermal conductivity are all dependent on temperature. Under Boussinesq approximation, the equation of state is given by

$$
\rho=\rho_{0}\left[1-\beta_{T}\left(T-T_{0}\right)-\beta_{C}\left(C-C_{0}\right)\right]
$$

The momentum equation, the energy balance equation and mass transfer equations governing the flow of viscous fluid with variable viscosity and thermal conductivity are given by 


$$
\begin{gathered}
\frac{d}{d Y}\left(\mu \frac{d U}{d Y}\right)+\rho_{0} g \beta_{T}\left(T-T_{0}\right)+\rho_{0} g \beta_{C}\left(C-C_{0}\right)-\frac{\partial p}{\partial X}=0 \\
\frac{d}{d Y}\left(K \frac{d T}{d Y}\right)+\mu\left(\frac{d U}{d Y}\right)^{2} \pm Q\left(T-T_{0}\right)=0 \\
D \frac{d^{2} C}{d Y^{2}}-\gamma\left(C-C_{0}\right)=0
\end{gathered}
$$

where $T_{0}$ is the reference temperature.

The boundary conditions for velocity, temperature and concentration are given by

$$
\begin{aligned}
& U=0, T=T_{1}, C=C_{1} \text { at } Y=-b \\
& U=0, T=T_{2}, C=C_{2} \text { at } Y=b
\end{aligned}
$$

The viscosity of the fluid is assumed to vary exponentially with temperature as considered in [14] and [15]

$$
\mu=\mu_{0} e^{-a\left(T-T_{0}\right)}=\mu_{0}\left(1-a\left(T-T_{0}\right)\right)
$$

where $a$ is empirical constant for viscosity.

The thermal conductivity of the fluid is also assumed to vary exponentially with temperature as in [15]

$$
K=K_{0} e^{-\tilde{b}\left(T-T_{0}\right)}=K_{0}\left(1-\tilde{b}\left(T-T_{0}\right)\right)
$$

where $\tilde{b}$ is empirical constant for thermal conductivity.

The dimensionless parameters used to write the non-dimensional forms of the governing equations (2)-(4) are

$$
\begin{gathered}
u=\frac{U}{\bar{u}}, \quad y=\frac{Y}{b}, \quad m=\frac{T_{1}-T_{2}}{\Delta T}, n=\frac{C_{1}-C_{2}}{\Delta C}, \phi=\frac{C-C_{0}}{\Delta C}, \theta=\frac{T-T_{0}}{\Delta T}, \\
\lambda_{T}=\frac{G r_{T}}{\operatorname{Re}}, \alpha=\frac{\gamma b^{2}}{D}, P=\frac{b^{2}}{\mu_{0} \bar{u}} \frac{\partial p}{\partial x}, \lambda_{C}=\frac{G r_{C}}{\operatorname{Re}}, G r_{T}=\frac{g \beta_{T} b^{3} \Delta T}{v^{2}}, \\
G r_{C}=\frac{g \beta_{C} b^{3} \Delta C}{v^{2}}, B r=\frac{\mu_{0} \bar{u}^{2}}{K_{0} \Delta T}, \operatorname{Re}=\frac{\bar{u} b}{v}
\end{gathered}
$$

Using these dimensionless parameters, the governing equations (2) to (4) takes the form 


$$
\begin{gathered}
\frac{d^{2} u}{d y^{2}}=-b_{v} \frac{d \theta}{d y} \frac{d u}{d y}+\left(1+b_{v} \theta\right)\left(\lambda_{T} \theta+\lambda_{C} \phi\right)-\left(1+b_{v} \theta\right) P \\
\frac{d^{2} \theta}{d y^{2}}-b_{k}\left(\frac{d \theta}{d y}\right)^{2}+B r\left(\frac{d u}{d y}\right)^{2}-\left(b_{k}-b_{v}\right) \theta\left(\frac{d u}{d y}\right)^{2}-b_{k} b_{v} \theta^{2} B r\left(\frac{d u}{d y}\right)^{2} \pm \psi\left(\theta+b_{k} \theta^{2}\right)=0 \\
\frac{d^{2} \phi}{d y^{2}}-\alpha \phi=0
\end{gathered}
$$

The boundary conditions in dimensionless form can be written as

$$
\begin{gathered}
u=0, \theta=1+m, \phi=1+n \text { at } y=-1 \\
u=0, \theta=1, \phi=1 \text { at } y=1
\end{gathered}
$$

where $\Delta T=T_{2}-T_{0}, \Delta C=C_{2}-C_{0}, b_{v}=a \Delta T$ is the variable viscosity parameter, $b_{k}=\tilde{b} \Delta T$ is the variable conductivity parameter, $m=\frac{T_{1}-T_{2}}{\Delta T}$ is the wall temperature ratio, $n=\frac{C_{1}-C_{2}}{\Delta C}$ is the wall concentration ratio.

\section{Solution}

The governing nonlinear coupled equations are solved numerically using Runge-Kutta fourth order shooting method. The effects of viscosity variation parameter $b_{v}$, thermal conductivity variation parameter $b_{k}$, concentration parameter $\alpha$ and heat generation parameter $\psi$ are all studied. Velocity, temperature, concentration profiles are presented graphically and analyzed.

Equation (12) can be solved analytically and the solution is given by

$$
\phi=C_{1} \operatorname{Cosh}(\sqrt{\alpha}) y+C_{2} \operatorname{Sinh}(\sqrt{\alpha}) y
$$

Then, equations (10) and (11) are solved using Runge-Kutta 4th order method. For this method to apply, we must have system of first order differential equations with initial conditions. We use shooting technique for guessing the initial conditions and then solving these equations using Runge-Kutta 4th order method formula.

For, $\frac{d y}{d x}=f(x, y)$ with $y\left(x_{0}\right)=y_{0}$

$$
y(x)=\frac{1}{6}\left(k_{1}+2 k_{2}+2 k_{3}+k_{4}\right)
$$


where:

$k_{1}=h f\left(x_{0}, y_{0}\right)$

$k_{2}=h f\left(x_{0}+\frac{h}{2}, y_{0}+\frac{k_{1}}{2}\right)$

$k_{3}=h f\left(x_{0}+\frac{h}{2}, y_{0}+\frac{k_{2}}{2}\right)$

$k_{4}=h f\left(x_{0}+h, y_{0}+k_{3}\right)$

Further, along with velocity, temperature and concentration, the physical quantities like shear stress and Nusselt numbers can be analysed. Skin friction and Nusselt numbers at the boundary walls of the channel as

$$
\begin{gathered}
\tau_{1}=e^{-b_{v}(1+m)}\left(\frac{d u}{d y}\right)_{y=-1} \text { and } \tau_{2}=e^{-b_{v}}\left(\frac{d u}{d y}\right)_{y=1} \\
N u_{1}=-\left(1+b_{k}(1+m)\right)\left(\frac{d \theta}{d y}\right)_{y=-1} \text { and } N u_{2}=-\left(1+b_{k}\right)\left(\frac{d \theta}{d y}\right)_{y=1}
\end{gathered}
$$

\section{Results and discussion}

The flow and heat transfer in a vertical channel with variable viscosity and thermal conductivity with first order chemical reaction and heat generation / absorption is studied. The obtained governing equations are non-linear and coupled. The coupled non-linear equations are solved numerically by using the Runge-Kutta fourth order along with the shooting technique. The solutions are obtained and analysed graphically for various physical parameters such as viscosity parameter $b_{v}$, thermal conductivity parameter $b_{k}$, and heat generation/absorption parameter $\psi$.

When the heat generation/absorption is not considered in the flow phenomena, then the effects of thermal buoyancy parameter $\mathrm{Gr}_{\mathrm{t}}$, Brinkman number $\mathrm{Br}$, temperature ratio $m$, chemical reaction term $\alpha$ on velocity and temperature profiles are exactly matches with the result obtained by Umavathi [20].
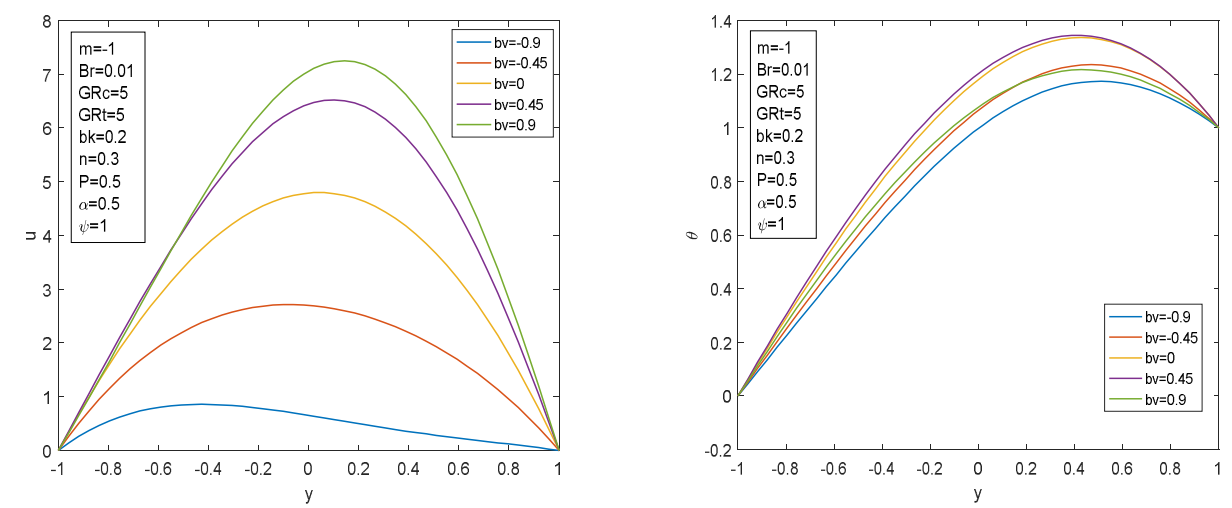

Fig. 2. Velocity profile for different values of $b_{v}$ Fig. 3. Temperature profile for different values of $b_{v}$ 
Figures 2 and 3 show the effect of viscosity variation parameter $b_{v}=-0.9,-0.45$, $0,0.45,0.9$ on velocity and temperature profiles for a constant thermal conductivity. These figures show that with increase in the value of viscosity variation parameter, both velocity and temperature profile increases. It can be seen from the Figure 2 that for negative values of $b_{v}$ velocity profiles are shifted towards the cold wall and for positive values of $b_{v}$ velocity profiles are shifted towards the hot wall. When $b_{v}$ increases, the temperature of the fluid increases due to heat generation by the internal friction caused by the collision of the fluid particles.
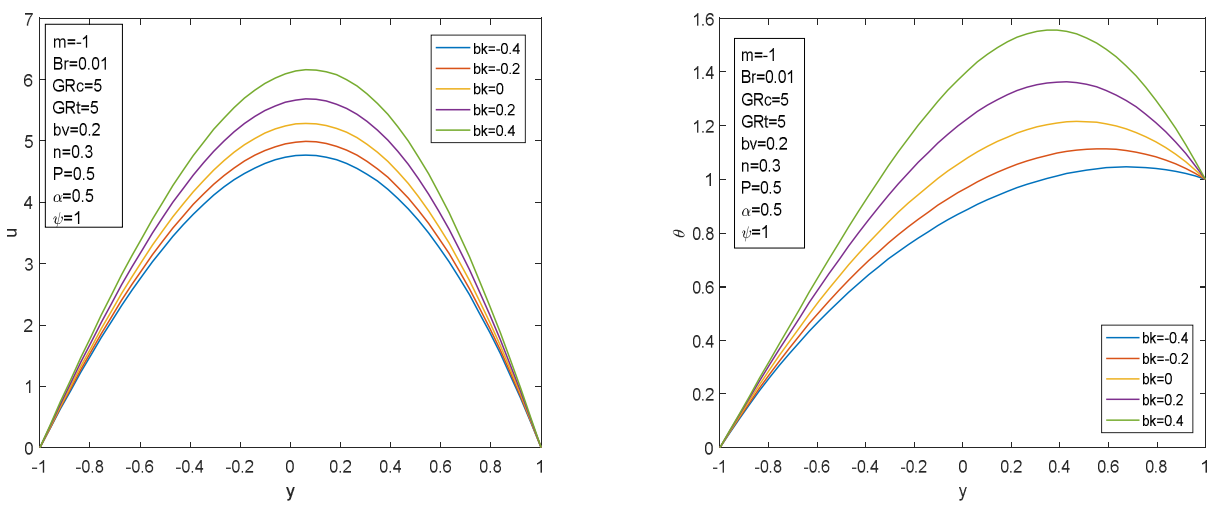

Fig. 4. Velocity profile for different values of $b_{k}$ Fig. 5. Temperature profile for different values of $b_{k}$

Figures 4 and 5 represents the effect of thermal conductivity variation parameter $b_{k}=-0.4,-0.2,0,0.2,0.4$ on velocity and temperature profiles for a constant viscosity. We observe from these two graphs an increase in the value of $b_{k}$ which enhances the velocity and temperature profiles. The velocity profile is parabolic and the magnitude is high in middle of the channel due to thermal conductivity. The temperature profile clearly indicates that the magnitude is high at the hot wall where as it is low at the cold wall.
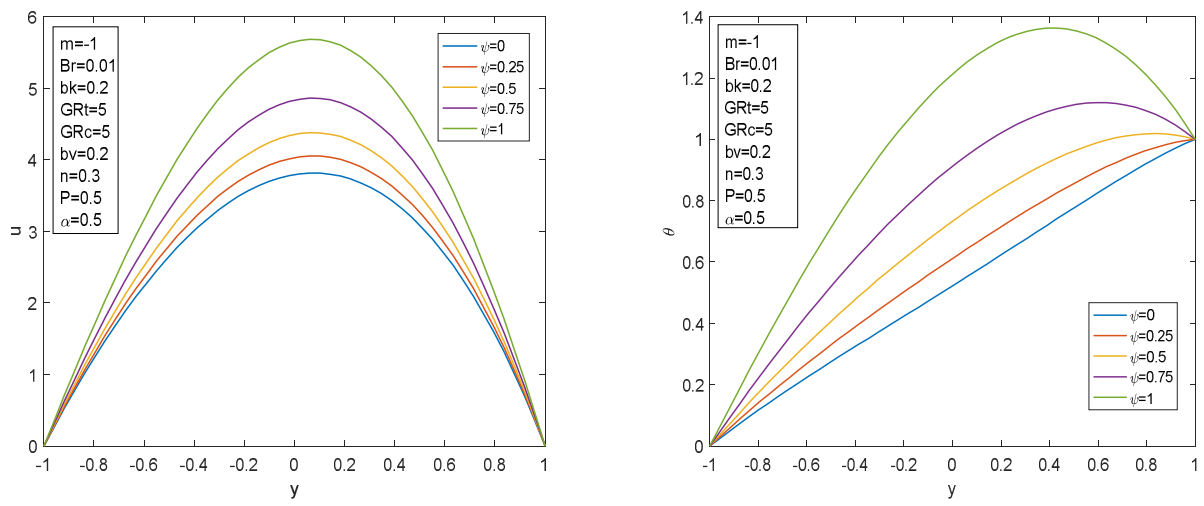

Fig. 6. Velocity profile for different values of $\psi$ Fig. 7. Temperature profile for different values of $\psi$ 
The effect of heat generation parameter $\psi=0.0,0.25,0.5,0.75,1.0$ on velocity and temperature are presented in Figures 6 and 7. It can be observed that with an increase in the value of the heat generation parameter both, velocity and temperature increase.
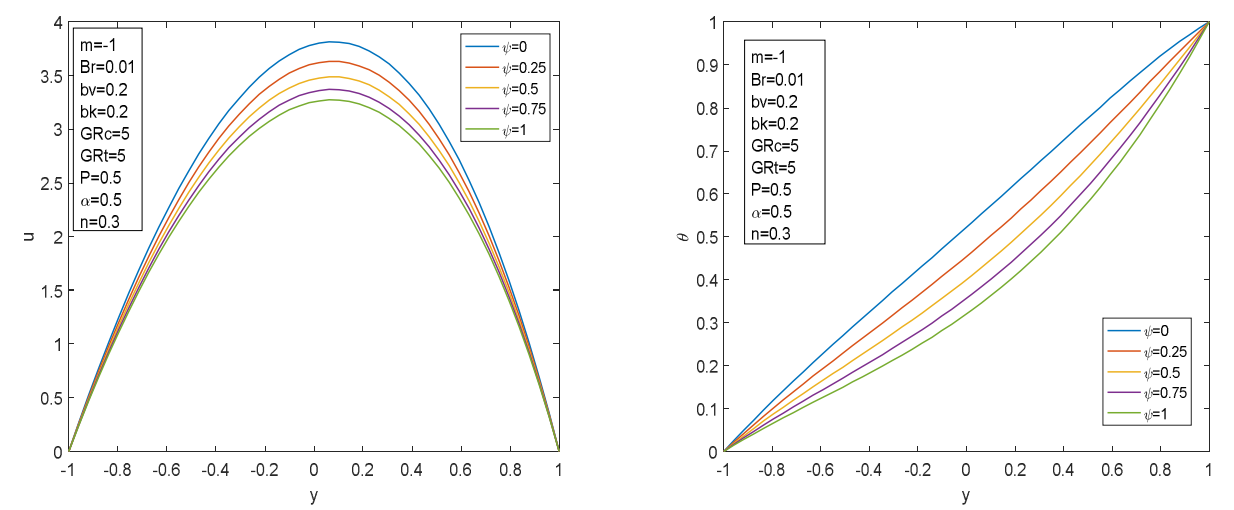

Fig. 8. Velocity profile for different values of $\psi$ Fig. 9. Temperature profile for different values of $\psi$

Table 1. Values of Skin friction and Nusselt number for different physical parameters

\begin{tabular}{|c|c|c|c|c|c|}
\hline & $\tau_{1}$ & $\tau_{2}$ & & $\mathrm{Nu}_{1}$ & $\mathrm{Nu}_{2}$ \\
\hline & \multicolumn{5}{|c|}{$b_{k}=0.2, m=-1, \mathrm{Br}=0.01, \mathrm{GR}_{\mathrm{C}}=5, \mathrm{GR}_{\mathrm{T}}=5, \alpha=0.5, n=0.3, P=0.5, \psi=1$} \\
\hline$b_{v}$ & \multicolumn{2}{|c|}{$b_{k}=0.2$} & $b_{k}$ & \multicolumn{2}{|c|}{$b_{v}=0.2$} \\
\hline-0.9 & 21.7334 & 3.4749 & -0.4 & -1.4128 & 0.1834 \\
\hline-0.5 & 17.2325 & -8.1339 & -0.2 & -1.4501 & 0.4486 \\
\hline 0.0 & 8.4941 & -9.9298 & 0.0 & -1.4972 & 0.8776 \\
\hline 0.5 & 3.1682 & -8.0589 & 0.2 & -1.5489 & 1.5129 \\
\hline 0.9 & 1.2949 & -6.1156 & 0.4 & -1.5782 & 2.3368 \\
\hline M & \multicolumn{5}{|c|}{$b_{k}=0.2, b_{v}=0.2, \mathrm{Br}=0.01, \mathrm{GR}_{\mathrm{C}}=5, \mathrm{GR}_{\mathrm{T}}=5, \alpha=0.5, n=0.3, P=0.5, \psi=1$} \\
\hline-2 & 2.7570 & -5.2372 & & -0.4003 & -0.9829 \\
\hline-1 & 8.8103 & -9.4563 & & -1.5489 & 1.5129 \\
\hline 0 & 15.8940 & -15.4093 & & -5.0994 & 5.0587 \\
\hline 1 & 19.2522 & -18.8275 & & -8.6655 & 7.0658 \\
\hline 2 & 20.1770 & -20.3077 & & -10.6251 & 7.7829 \\
\hline$\psi(\mathrm{Gen})$ & \multicolumn{5}{|c|}{$b_{k}=0.2, b_{v}=0.2, \mathrm{Br}=0.01, \mathrm{GR}_{\mathrm{C}}=5, \mathrm{GR}_{\mathrm{T}}=5, \alpha=0.5, n=0.3, P=0.5, m=-1$} \\
\hline 0 & 6.6681 & -7.3437 & & -0.61777 & -0.3985 \\
\hline 0.25 & 6.9528 & -7.6350 & & -0.73772 & -0.0977 \\
\hline 0.5 & 7.3385 & -8.0225 & & -0.90302 & 0.2760 \\
\hline 0.75 & 7.8979 & -8.5737 & & -1.1464 & 0.7726 \\
\hline 1 & 8.8103 & -9.4563 & & -1.5489 & 1.5129 \\
\hline$\psi(\mathrm{Abs})$ & \multicolumn{5}{|c|}{$b_{k}=0.2, b_{v}=0.2, \mathrm{Br}=0.01, \mathrm{GR}_{\mathrm{C}}=5, \mathrm{GR}_{\mathrm{T}}=5, \alpha=0.5, n=0.3, P=0.5, m=-1$} \\
\hline 0 & 6.6681 & -7.3437 & & 0.61777 & -0.3985 \\
\hline 0.25 & 6.4484 & -7.1147 & & 0.52688 & -0.6510 \\
\hline 0.5 & 6.2732 & -6.9289 & & 0.45584 & -0.8695 \\
\hline 0.75 & 6.13 & -6.7741 & & 0.399 & -1.0628 \\
\hline 1 & 6.0108 & -6.6430 & & 0.35268 & -1.2369 \\
\hline
\end{tabular}


Figures 8 and 9 represent the effect of heat absorption parameter $\psi=0.0,0.25$, $0.5,0.75,1.0$ on velocity and temperature profiles. It can be observed that with increase in the value of the heat absorption parameter, both velocity and temperature decrease.

The effect of different parameters such as variable viscosity, variable thermal conductivity, wall temperature ratio and heat generation / absorption parameters on skin friction and Nusselt numbers at both the boundaries are shown in Table 1.

We can observe that with increase in the value of variable viscosity parameter $b_{v}$, skin friction decreases at both of the boundary walls, but with an increase in the value of variable thermal conductivity parameter $b_{k}$, the magnitude of the Nusselt number increases at the boundary walls. The same effects can be observed with the variation of wall temperature ratio parameter. As the heat generation parameter increases, the magnitude of skin friction at both the boundary walls increases. Nusselt number also increases in magnitude. The exact opposite effect can be observed with heat absorption.

Table 2 shows the values of skin friction and Nusselt number at left wall of the channel for different values of $b_{v}$ obtained in the present work and work done by Umavathi [20]. These values show that the results obtained in this work are very much compatible with the published results. Hence, validation of results can be observed.

Table 2. Comparison of present work with published work by Umavathi [20] on skin friction and Nusselt number values at the left wall of the channel

\begin{tabular}{|c|c|c|c|c|}
\hline & \multicolumn{2}{|c|}{$\tau_{\mathbf{1}}$} & \multicolumn{2}{c|}{$\mathrm{Nu}_{\mathbf{1}}$} \\
\hline & \multicolumn{2}{|c|}{$b_{k}=0.2, m=-1, \mathrm{Br}=0.01, \mathrm{GR}_{\mathrm{C}}=5, \mathrm{GR}_{\mathrm{T}}=5, \alpha=0.5, n=0.3, P=0.5, \psi=0$} \\
\hline$b_{v}$ & Present Work & Umavathi et al. & Present Work & Umavathi et al. \\
\hline-0.9 & 4.8395 & 4.8370 & -0.4856 & -0.4780 \\
-0.5 & 5.7502 & 5.6230 & -0.5170 & -0.5093 \\
0.0 & 6.4439 & 6.4134 & -0.5816 & -0.5784 \\
0.5 & 6.8682 & 7.0006 & -0.7278 & -0.7039 \\
0.9 & 7.0126 & 7.2836 & -0.8976 & -0.8896 \\
\hline
\end{tabular}

\section{Conclusions}

In this paper, we have studied the effect of heat generation/ absorption on mixed convective flow of viscous fluid in a vertical channel with variable viscosity and thermal conductivity. The governing equations are solved using the Runge-Kutta $4^{\text {th }}$ order shooting method and analyzed the effects of different physical parameters on velocity and temperature fields graphically. We can draw the following results:

1. Variable viscosity and variable thermal conductivity increases the velocity and temperature of the flow. 
2. Due to increased molecular collision in the flow field, concentration buoyancy parameter enhances the flow and heat transfer.

3. The presence of heat generation enhances the flow and heat transfer because there is an increase of internal heat which result in the rise of flow field, whereas heat absorption suppresses the flow and heat transfer.

\section{Nomenclature}

a empirical constant for the viscosity

$b \quad$ characteristic length of the channel [m]

$\tilde{b}$ empirical constant for thermal conductivity

$b_{v} \quad$ viscosity variation parameter

$b_{k} \quad$ conductivity variation parameter

$\mathrm{Br} \quad$ Brinkman number

$C_{1}, C_{2}$ concentrations along the walls

$C_{0} \quad$ reference species concentration

C concentration of the fluid

$\mathrm{GR}_{\mathrm{T}}$ modified thermal Grashof number

$\mathrm{Gr}_{\mathrm{T}}$ thermal Grashof number

$\mathrm{GR}_{\mathrm{C}}$ modified mass Grashof number

$\mathrm{Gr}_{\mathrm{C}}$ mass Grashof number

Re Reynolds number

$g \quad$ acceleration due to gravity $\left[\mathrm{ms}^{-2}\right]$

$K \quad$ thermal conductivity of the fluid [W/m K]

$K_{0} \quad$ thermal conductivity at temperature $T_{0}[\mathrm{~W} / \mathrm{m} \mathrm{K}]$

$m \quad$ wall temperature ratio

$n \quad$ wall concentration ratio

$T_{0} \quad$ reference temperature [K]

$T$ fluid temperature [K]

$T_{1}, T_{2}$ temperatures along the walls [K]

$U \quad$ velocity $\left[\mathrm{ms}^{-1}\right]$

$u \quad$ dimensionless velocity

$\bar{u} \quad$ mean velocity

$Y \quad$ dimensional coordinate axis [m]

$y \quad$ dimensionless coordinate axis

\section{Greek letters}

$\alpha$ dimensionless chemical reaction parameter

$\beta_{T}$ coefficient of thermal expansion $\left[\mathrm{K}^{-1}\right]$ 


$\begin{array}{ll}\beta_{C} & \text { concentration expansion coefficient }\left[\mathrm{K}^{-1}\right] \\ \gamma & \text { chemical reaction parameter } \\ \theta & \text { dimensionless temperature } \\ \mu & \text { dynamic viscosity }[\mathrm{Pa} \mathrm{s}] \\ \mu_{0} & \text { dynamic viscosity at temperature } T_{0}[\mathrm{~Pa} \mathrm{~s}] \\ v & \text { kinematic viscosity }\left[\mathrm{m} \mathrm{s}^{2}\right] \\ \rho & \text { density of the fluid }\left[\mathrm{kg} \mathrm{m}^{-3}\right] \\ \rho_{0} & \text { static density }\left[\mathrm{kg} \mathrm{m}^{-3}\right] \\ \phi & \text { rescaled species concentration } \\ \psi & \text { heat generation/absorption parameter } \\ \tau_{1}, \tau_{2} & \text { skin friction }\end{array}$

\section{References}

[1] Bejan, A. (2013). Convection Heat Transfer. New Jersey, Wiley, Breckling J., The Analysis of Directional Time Series: Applications to Wind Speed and Direction, ser. Lecture Notes in Statistics. Berlin: Springer, 61.

[2] Umavathi, J.C. (2011). Free convection of composite porous medium in a vertical channel. Heat Transfer-Asian Research, 40(4), 308-329.

[3] Muthucumaraswamy, R., \& Ganesan, P. (2001). First-order chemical reaction on flow past an impulsively started vertical plate with uniform heat and mass flux. Acta Mechanica, 147, 45-57.

[4] Umavathi, J.C., Patil Mallikarjun, B., \& Pop, I. (2006). On laminar mixed convection flow in a vertical porous stratum with asymmetric wall heating conditions. International Journal of Transport Phenomenon, 8(2).

[5] Seddeek, M.A. (2005). Finite-element method for the effects of chemical reaction, variable viscosity, thermophoresis and heat generation/absorption on a boundary-layer hydromagnetic flow with heat and mass transfer over a heat surface, Acta Mechanica, 177, 1-18.

[6] Herwig, H., \& Wickern, G. (1986). The effect of variable properties on laminar boundary layer flow. Wärme- und Stoffübertragung, 20, 47-57.

[7] Pop, I., Gorla, R.S.R., \& Rashidi, M. (1992). The effect of variable viscosity on flow and heat transfer to a continuous moving flat plate. Int. J. Eng. Sci., 30(1), 1-6.

[8] Chiam, T.C. (1996). Heat transfer with variable thermal conductivity in a stagnation-point flow towards a stretching sheet. Int. Comm. Heat Mass Transfer, 23, 239-248.

[9] Abel, M.S., Khan, S.K., \& Prasad, K.V. (2002). Study of visco-elastic fluid flow and heat transfer over a stretching sheet with variable viscosity. Int. J. Non-Linear Mech., 37, 81-88.

[10] Gary, J., Kassoy, D.R., Tadjeran, H., \& Zebib A. (1982). The effects of significant viscosity variation on convective heat transport in water-saturated porous medium. J. Fluid Mech., 117, 233-249.

[11] Mehta, K.N., \& Sood, S. (1992). Transient free convection flow with temperature dependent viscosity in a fluid saturated porous medium. Int. J. Eng. Sci., 30, 1083-1087.

[12] Zamora, B., \& Hernández, J. (1997). Influence of variable property effects on natural convection flows in asymmetrically-heated vertical channels. Int. Comm. Heat Mass Transfer, 24, $1153-1162$. 
[13] Hernández, J., \& Zamora, B. (2005). Effects of variable properties and non-uniform heating on natural convection flows in vertical channels. International Journal Heat and Mass Transfer, 48, 793-807.

[14] Barletta, A., \& Zanchini, E. (1999). On the choice of the reference temperature for fully- developed mixed convection in a vertical channel. International Journal Heat and Mass Transfer, 42, 3169-3181.

[15] Attia, H.A. (2006). Unsteady hydromagnetic channel flow of dusty fluid with temperature dependent viscosity and thermal conductivity. Heat Mass Transfer, 42, 779-787.

[16] Patil Mallikarjun, B., Umavathi, J.C., \& Narashimha Murthy, S. (2013). On laminar magnetoconvection flow in a vertical channel in the presence of heat generation or heat absorption. International Journal of Heat Transfer, 135(4), 042503-1 to 042503-8.

[17] Chamka, A.J. (2003). MHD flow of a uniformly stretched vertical permeable surface in the presence of heat generation/absorption and a chemical reaction. Int. Comm. Heat Mass Transfer, 30, 413-422.

[18] Prathap Kumar, J., Umavathi, J.C., \& Jagtap Sharadkumar (2013). Effect of first order chemical reaction in a vertical double-passage channel. International Journal of Engineering Research and Applications, 3(5), 967-977.

[19] Patil Mallikarjun, B., \& Vasudeva Murthy, R. (2018). Finite element analysis of fully developed mixed convection through a vertical channel in the presence of heat generation/absorption with fist order chemical reaction. Defect and Diffusion Forum, 388, 394-406.

[20] Umavathi, J.C. (2016). Combined effect of variable viscosity and thermal conductivity on mixed convection flow of a viscous fluid in a vertical channel in the presence of first order chemical reaction. European Journal of Mechanics B/Fluids, 58, 98-118. 